\title{
DIFFERENTIAL AS A HARMONIC MORPHISM WITH RESPECT TO CHEEGER-GROMOLL TYPE METRICS
}

\author{
W. KOZEOWSKI AND K. NIEDZIALOMSKI
}

\begin{abstract}
We investigate horizontal conformality of a differential of a map between Riemannian manifolds where the tangent bundles are equipped with Cheeger-Gromoll type metrics. As a corollary, we characterize the differential of a map as a harmonic morphism.
\end{abstract}

\section{INTRODUCTION AND PRELIMINARY RESULTS}

1.1. Introduction. In [2], M. Benyounes, E. Loubeau and C. M. Wood intoduced a new class of Riemannian metrics in the tangent bundle over a Riemanian manifold. These metrics $h_{p, q}$, depending on two constants $p, q$, generalize Sasaki and Cheeger-Gromoll metrics. Wider class of this type of metrics was investigated by M. I. Munteanu [8] and by the authors [6]. Now, there are several articles concerning geometry of a tangent bundle equipped with Cheeger-Gromoll type metric. For example, M. Benyounes, E. Loubeau and C. M. Wood [3] considered $h_{p, q}$ metrics in the context of harmonic maps, whereas the first named author and Sz. M. Walczak [7] in the context of Riemanian submersions and Gromov-Hausdorff topology.

In [6], the authors studied the conformality of a differential $\Phi=\varphi_{*}$ : $(T M, \tilde{h}) \rightarrow(T N, h)$ of a map $\varphi: M \rightarrow N$ between Riemanian manifolds, where $\tilde{h}=h_{p, q, \alpha}$ and $h=h_{r, s, \beta}$ are metrics of Cheeger-Gromoll type. In this paper, we continue considerations concerning the differential of a map. We give necessary and sufficient conditions for a differential to be horizontally conformal and as a corollary we characterize the differential as a harmonic morphisms.

The idea is the following. The vertical part of Cheeger-Gromoll type metrics is nonlinear with respect to the base point, excluding Sasaki metric. Hence, conformal change of a metric on $M$ does not effect conformal change of Cheeger-Gromoll type metric. Scaling the base point, the condition of horizontal conformality reduces to vanishing of a certain polynomial, which

Date: November 17, 2018.

2000 Mathematics Subject Classification. 53C07; 53C43; 53A30.

Key words and phrases. Horizontally conformal mapping, harmonic morphism, tangent bundle, Cheeger-Gromoll type metric. 
gives restrictions to coefficients $p, q, r, s$ and $\alpha, \beta$. That is why, for horizontal conformality of $\Phi$ there are not many natural choices of $\tilde{h}$ and $h$ (Theorem 2.1), whereas in the context of a harmonic morphism the only possibility is Sasaki metric (Theorem 2.2).

1.2. Preliminary results. Let $(M, g)$ be a Riemannian manifold and $\pi$ : $T M \rightarrow M$ its tangent bundle. The Levi-Civita connection and the projection $\pi$ gives the natural splitting $T T M=\mathcal{H} \oplus \mathcal{V}$ of the second tangent bundle $\pi_{*}: T T M \rightarrow T M$, where the vertical distribution $\mathcal{V}$ is the kernel of $\pi_{*}$ and the horizontal distribution $\mathcal{H}$ is the kernel of the conection map $K$. If $X, \xi \in T_{x} M$ then there is a unique vertical vector $X_{\xi}^{v}$ and a unique horizontal vector $X_{\xi}^{h}$ in $T_{\xi} T M$ such that $\pi_{*} X_{\xi}^{h}=X$ and $K X_{\xi}^{v}=X$. Moreover, any $A \in T_{\xi} T M$ has a unique decomposition into horozontal and vertical part, $A=\mathcal{V} A+\mathcal{H} A$. For more details on decomposition of the tangent bundle see [5].

Let $p, q, \alpha$ be constants, $q$ non-negative, $\alpha$ positive. Define $(p, q, \alpha)$ metric $h=h_{p, q, \alpha}$ on $T M$ as follows. For every $A, B \in T_{\xi}(T M)$,

$$
h(A, B)=g\left(\pi_{*} A, \pi_{*} B\right)+\omega_{\alpha}(\xi)^{p}(g(K A, K B)+q g(K A, \xi) g(K B, \xi)),
$$

where $\omega_{\alpha}(\xi)=(1+\alpha g(\xi, \xi))^{-1}$. The Riemannian metric $h_{p, q, \alpha}$ is a generalization of the metric considered in [2, 3] and is a special case of a metric considered in [8]. In particular, $h_{0,0, \alpha}\left(\right.$ or $\left.h_{p, 0,0}\right)$ is Sasaki metric, $h_{1,1,1}$ Cheeger-Gromoll metric.

We will often write $\langle\cdot, \cdot\rangle_{M}$ for $g$ and $\langle\cdot, \cdot\rangle_{T M}$ for $h=h_{p, q, \alpha}$. The lenght of a vector will be denoted by $|\cdot|_{M}$ and $|\cdot|_{T M}$, respecitively.

Consider now a smooth map $\varphi: M \rightarrow N$ between Riemannian manifolds $M$ and $N$. Let $\varphi^{-1} T N \rightarrow M$ be a pull-back bundle. There is a unique connection $\nabla^{\varphi}$ in this bundle characterised by the property [1]

$$
\nabla_{X}^{\varphi}(Y \circ \varphi)=\nabla_{\varphi_{*} X}^{N} Y, \quad X \in T_{x} M, Y \in \Gamma(T N) .
$$

Then we easily obtain

$$
\nabla_{X}^{\varphi} \varphi_{*} Y-\nabla_{Y}^{\varphi} \varphi_{*} X=\varphi_{*}[X, Y], \quad X, Y \in \Gamma(T M) .
$$

The second fundamental form of $\varphi$ is $B=\nabla \varphi_{*}$,

$$
B(X, Y)=\nabla_{X}^{\varphi} \varphi_{*} Y-\varphi_{*}\left(\nabla_{X}^{M} Y\right), \quad X, Y \in \Gamma(T M) .
$$

By (1.1), we get that $B$ is symmetric and hence tensorial in both variables. If $B=0$ we say that $\varphi$ is totally geodesic. We will need the following lemma. 
Lemma 1.1. For $X, \xi \in T_{x} M$

$$
\begin{aligned}
\varphi_{* *} X_{\xi}^{v} & =\left(\varphi_{*} X\right)_{\varphi_{*} \xi}^{v}, \\
\varphi_{* *} X_{\xi}^{h} & =\left(\varphi_{*} X\right)_{\varphi_{*} \xi}^{h}+(B(X, \xi))_{\varphi_{*} \xi}^{v} .
\end{aligned}
$$

Proof. Follows by the definition of a connection map and vertical and horizontal distributions. Details are left to the reader.

Let $\varphi:\left(M,\langle\cdot, \cdot\rangle_{M}\right) \rightarrow\left(N,\langle\cdot, \cdot\rangle_{N}\right)$ be a smooth map between Riemannian manifolds, $\operatorname{dim} M>\operatorname{dim} N$. Let $\mathcal{V}^{\varphi}=\operatorname{ker} \varphi_{*}$ be the vertical distribution and $\mathcal{H}^{\varphi}=\left(\mathcal{V}^{\varphi}\right)^{\perp}$, the orthogonal complement of $\mathcal{H}^{\varphi}$ with respect to $\langle\cdot, \cdot\rangle_{M}$, the horozontal distribution, $T M=\mathcal{V}^{\varphi} \oplus \mathcal{H}^{\varphi}$. Each $X \in T_{x} M$ has therefore a unique decomposition

$$
X=X^{\top}+X^{\perp}
$$

into vertical and horizontal part.

We say that $\varphi$ is horizontally conformal if for every $x \in M$ either $\varphi_{* x}=0$ or $\varphi_{* x}: \mathcal{H}_{x}^{\varphi} \rightarrow T_{\varphi(x)} N$ is surjective and

$$
\left\langle\varphi_{* x} X, \varphi_{* x} Y\right\rangle_{N}=\lambda(x)\langle X, Y\rangle_{M}, \quad X, Y \in \mathcal{H}_{x}^{\varphi},
$$

where $\lambda(x)$ is positive. We call $\lambda$ the dilatation of $\varphi$. Let $C_{\varphi}$ denote the set of critical points i.e., points $x \in M$ such that $\varphi_{* x}=0$. One can easily prove

Lemma 1.2. For $\Phi=\varphi_{*}: T M \rightarrow T N$ we have $\pi\left(C_{\Phi}\right) \subset C_{\varphi}$.

Moreover, by Lemma 1.1 we have

Lemma 1.3. If a map $\varphi: M \rightarrow N$ is a submersion, i.e., surjective map of maximal rank, then so is $\Phi=\varphi_{*}: T M \rightarrow T N$.

Remark 1.4. By Lemmas 1.2 and 1.3, if $\varphi: M \rightarrow N$ and $\Phi=\varphi_{*}$ : $T M \rightarrow T N$ are horizontally conformal, then the differential of horizontally conformal submersion $\varphi: M \backslash C_{\varphi} \rightarrow N$ is a horizontaly conformal submersion $\Phi: T M \backslash \pi^{-1}\left(C_{\varphi}\right) \rightarrow T N$. Hence, throughout the paper, without loss of generality, we may assume that horizontaly conformal map $\varphi$ is a submersion i.e. the set of critical points is empty.

Theorem 1.5. Assume $\varphi: M \rightarrow N$ is a submersion. Let $\Phi=\varphi_{*}: T M \rightarrow$ $T N$ and equip tangent bundles $T M$ and $T N$ with Cheeger-Gromoll type metrics $h_{p, q, \alpha}$ and $h_{r, s, \beta}$, respectively. Then, with respect to submersion $\Phi$, the second tangent bundle splits into orthogonal sum $T_{\xi} T M=\mathcal{V}_{\xi}^{\Phi} \oplus \mathcal{H}_{\xi}^{\Phi}$, where

$$
\mathcal{V}_{\xi}^{\Phi}=\operatorname{Span}\left\{\eta_{\xi}^{h}+\left(\nabla_{\xi}^{M} \eta\right)_{\xi}^{v} \mid \eta \in \Gamma\left(\mathcal{V}^{\varphi}\right)\right\}
$$


and

$$
\mathcal{H}_{\xi}^{\Phi}=\operatorname{Span}\left\{X_{\xi}^{v}-q \omega_{q}(\xi)\langle X, \xi\rangle \xi_{\xi}^{v}+\omega_{\alpha}(\xi)^{p}\left(\nabla_{\xi}^{M} X\right)_{\xi}^{h} \mid X \in \Gamma\left(\mathcal{H}^{\varphi}\right)\right\} .
$$

Proof. Let $\varphi:\left(M, g_{M}\right) \rightarrow\left(N, g_{N}\right)$ be a submersion. Fix $\xi \in T_{x} M, x \in M$. Denote the right hand side of (1.5) by $\mathbb{V}$ and the right hand side of (1.6) by $\mathbb{H}$. By Lemma 1.1, for $\eta \in \Gamma\left(\mathcal{V}^{\varphi}\right)$

$$
\Phi_{*}\left(\eta_{\xi}^{h}+\left(\nabla_{\xi}^{M} \eta\right)_{\xi}^{v}\right)=\left(\varphi_{*} \eta\right)_{\varphi_{*} \xi}^{h}+B(\eta, \xi)_{\varphi_{*} \xi}^{v}+\left(\varphi_{*} \nabla_{\xi}^{M} \eta\right)_{\varphi_{*} \xi}^{v}=0 .
$$

Hence, $\mathbb{V} \subset \mathcal{V}_{\xi}^{\Phi}$. Let $f$ be a smooth function such that $\xi f=1$ and $f(x)=0$. Then

$$
\mathcal{V}_{\xi}^{\Phi} \ni(f \eta)_{\xi}^{h}+\left(\nabla_{\xi}^{M} f \eta\right)_{\xi}^{v}=\eta_{\xi}^{v} .
$$

Therefore, we see that $\operatorname{dim} \mathbb{V}=2(m-n)$, where $m=\operatorname{dim} M$ and $n=\operatorname{dim} N$. Thus $\mathcal{V}_{\xi}^{\Phi}=\mathbb{V}$, so (1.5) holds. Now, let $\eta \in \Gamma\left(\mathcal{V}^{\varphi}\right)$ and $X \in \Gamma\left(\mathcal{H}^{\varphi}\right)$. For $A=\eta_{\xi}^{h}+\left(\nabla_{\xi}^{M} \eta\right)_{\xi}^{v}$ and $B=X_{\xi}^{v}-q \omega_{q}(\xi)\langle X, \xi\rangle \xi_{\xi}^{v}+\omega_{\alpha}(\xi)^{p}\left(\nabla_{\xi}^{M} X\right)_{\xi}^{h}$

$$
\begin{aligned}
\langle A, B\rangle_{T M} & =\omega_{\alpha}(\xi)^{p}\left(\left\langle X, \nabla_{\xi}^{M} \eta\right\rangle_{M}+q\langle X, \xi\rangle_{M}\left\langle\xi, \nabla_{\xi}^{M} \eta\right\rangle_{M}\right. \\
& -q \omega_{q}(\xi)\langle X, \xi\rangle_{M}\left\langle\nabla_{\xi}^{M} \eta, \xi\right\rangle_{M} \\
& \left.-q^{2} \omega_{q}(\xi)\langle X, \xi\rangle_{M}\left\langle\nabla_{\xi}^{M} \eta, \xi\right\rangle_{M}|\xi|_{M}^{2}+\left\langle\nabla_{\xi}^{M} X, \eta\right\rangle_{M}\right) \\
& =q \omega_{\alpha}(\xi)^{p}\langle X, \xi\rangle_{M}\left\langle\nabla_{\xi}^{M} \eta, \xi\right\rangle_{M}\left(1-\omega_{q}(\xi)-q \omega_{q}(\xi)|\xi|_{M}^{2}\right) \\
& =0 .
\end{aligned}
$$

Thus $\mathbb{H}$ is orthogonal to $\mathcal{V}_{\xi}^{\Phi}$. Again, as above, taking a function $f$ such that $\xi f=1$ and $f(x)=0$, we get $X_{\xi}^{h} \in \mathbb{H}$ for $X \in \Gamma\left(\mathcal{H}^{\varphi}\right)$. Hence, $\operatorname{dim} \mathbb{H}=2 m$. Therefore, $\mathbb{H}=\mathcal{H}_{\xi}^{\Phi}$ and (1.6) holds.

The proof of Theorem 1.5 also implies

Corollary 1.6. Assume $\varphi: M \rightarrow N$ is a submersion. Let $\Phi=\varphi_{*}: T M \rightarrow$ $T N$ and equip tangent bundles $T M$ and $T N$ with Cheeger-Gromoll type metrics $h_{p, q, \alpha}$ and $h_{r, s, \beta}$, respectively. Then

$$
\begin{aligned}
\eta_{\xi}^{v} & \in \mathcal{V}_{\xi}^{\Phi} \quad \text { for } \eta \in \mathcal{V}^{\varphi}, \\
X_{\xi}^{h} & \in \mathcal{H}_{\xi}^{\Phi} \quad \text { for } X \in \mathcal{H}^{\varphi} .
\end{aligned}
$$

Let $\varphi: M \rightarrow N$, be a Riemannian submersion, $\Phi=\varphi_{*}: T M \rightarrow T N$. A vector field $\hat{Z} \in \Gamma\left(\mathcal{H}^{\varphi}\right)$ is called basic if there is a vector field $Z \in \Gamma(T N)$ such that $\varphi_{*} \hat{Z}=Z \circ \varphi$. The correspondence $Z \mapsto \hat{Z}$ is one-to-one, since $\varphi_{* x}: \mathcal{H}_{x}^{\varphi} \rightarrow T_{\varphi(x)} N$ is an isomorphisms. Let $T: \Gamma\left(\mathcal{H}^{\varphi}\right) \times \Gamma\left(\mathcal{H}^{\varphi}\right) \rightarrow \Gamma\left(\mathcal{V}^{\varphi}\right)$ be an itegrability tensor of $\varphi$,

$$
T(X, Y)=\frac{1}{2}[X, Y]^{\top} .
$$


Let $\nabla^{M}$ and $\nabla^{N}$ be the Levi-Civita conections of $\langle\cdot, \cdot\rangle_{M}$ and $\langle\cdot, \cdot\rangle_{N}$, respectively. Then one can prove that for basic vector fields $\hat{Z}, \hat{W}$ and vertical vector field $\xi$

$$
-\left\langle\left(\nabla_{\xi}^{M} \hat{Z}\right)^{\perp}, \hat{W}\right\rangle_{M}=\langle\xi, T(\hat{Z}, \hat{W})\rangle_{M} .
$$

For more details on Riemannian submersions see [4, Chapter 9].

Assume now, $\varphi:\left(M,\langle\cdot, \cdot\rangle_{M}\right) \rightarrow\left(N,\langle\cdot, \cdot\rangle_{N}\right)$ is a horizontally conformal map with a dilatation $\lambda$. If we put $\langle\cdot, \cdot\rangle_{\lambda}=\lambda\langle\cdot, \cdot\rangle_{M}$, then $\varphi:\left(M,\langle\cdot, \cdot\rangle_{\lambda}\right) \rightarrow$ $\left(N,\langle\cdot, \cdot\rangle_{N}\right)$ is a Riemannian submersion. Clearly, horizontal and vertical distrbutions with respect to $\langle\cdot, \cdot\rangle_{M}$ and $\langle\cdot, \cdot\rangle_{\lambda}$ coincide. The Levi-Civita connections $\nabla^{M}$ and $\nabla^{\lambda}$ of $\langle\cdot, \cdot\rangle_{M}$ and $\langle\cdot, \cdot\rangle_{\lambda}$ satisfy

$$
\nabla_{X}^{\lambda} Y=\nabla_{X}^{M} Y+S(X, Y)
$$

where $S$ is a symmetric tensor field given by

$$
S(X, Y)=\frac{1}{2 \lambda}\left((X \lambda) Y+(Y \lambda) X-\langle X, Y\rangle_{M} \operatorname{grad} \lambda\right) .
$$

Let $\Phi=\varphi_{*}: T M \rightarrow T N$ and equip $T M$ and $T N$ with Cheeger-Gromoll type metrics $h_{p, q}$ and $h_{r, s}$, respectively. For simplicity, put

$$
P(X, \xi)=\sum_{i=1}^{n}\left\langle\xi, T\left(X, e_{i}\right)\right\rangle_{M} \varphi_{*} e_{i}, \quad \xi \in T_{x} M, X \in \mathcal{H}_{x}^{\varphi},
$$

where $e_{1}, \ldots, e_{n}$ is an orthonormal basis of $\mathcal{H}_{x}^{\varphi}$.

Lemma 1.7. Let $X \in \mathcal{H}_{x}^{\varphi}, \xi \in T_{x} M, x \in M$. Then

$$
\Phi_{*}\left(X_{\xi}^{h}\right)=\left(\varphi_{*} X\right)_{\varphi_{*} \xi}^{h}+\left(\varphi_{*} S(X, \xi)\right)_{\varphi_{*} \xi}^{v}+P(X, \xi)_{\varphi_{*} \xi}^{v} .
$$

Proof. We may assume that $X_{\hat{\zeta}}^{h}=\left(\gamma^{h}\right)^{\cdot}(0)$, where $\gamma$ is a curve on $M$ such that $\gamma(0)=x, \dot{\gamma}(t) \in \mathcal{H}^{\varphi}$ and $\dot{\gamma}(0)=X$, and $\gamma^{h}$ is a horizontal lift of $\gamma$ to $T M$ such that $\gamma^{h}(0)=\xi$. Put $\eta=\gamma^{h}$ for simplicity. Then

$$
\nabla_{\varphi_{*} \dot{\gamma}}^{N} \varphi_{*} \eta=\nabla_{\varphi_{*} \dot{\gamma}}^{N} \varphi_{*} \eta^{\perp}=\varphi_{*}\left(\nabla_{\dot{\gamma}}^{\lambda} \eta^{\perp}\right)
$$

and by (1.10) and the fact that $\eta$ is parallel with respect to $\nabla^{M}$,

$$
\nabla_{\dot{\gamma}}^{\lambda} \eta=S(\dot{\gamma}, \eta)
$$

Therefore,

$$
\nabla_{\dot{\gamma}}^{\lambda} \eta^{\perp}=S(\dot{\gamma}, \eta)-\nabla_{\dot{\gamma}}^{\lambda} \eta^{\top}
$$

Next, for a vector field $Y$ on $N$, by (1.9)

$$
\left\langle\varphi_{*}\left(\nabla_{\dot{\gamma}}^{\lambda} \eta^{\top}\right), Y\right\rangle_{N}=\lambda\left\langle\nabla_{\dot{\gamma}}^{\lambda} \eta^{\top}, \hat{Y}\right\rangle_{M}=\lambda\left\langle\nabla_{\eta^{\top}}^{\lambda} \dot{\gamma}, \hat{Y}\right\rangle_{M}=-\lambda\left\langle\eta^{\top}, T(\dot{\gamma}, \hat{Y})\right\rangle_{M} .
$$

Thus

$$
\varphi_{*}\left(\nabla_{\dot{\gamma}}^{\lambda} \eta^{\top}\right)=\frac{1}{\lambda} \sum_{i}\left\langle\varphi_{*}\left(\nabla_{\dot{\gamma}}^{\lambda} \eta^{\top}\right), \varphi_{*} e_{i}\right\rangle_{N} \varphi_{*} e_{i}=-\lambda \sum_{i}\left\langle\eta^{\top}, T\left(\dot{\gamma}, e_{i}\right)\right\rangle_{M} \varphi_{*} e_{i} .
$$


Since

$$
\begin{aligned}
K\left(\Phi_{*} X_{\xi}^{h}\right) & =\nabla_{\varphi_{*} X}^{N} \varphi_{*} \eta \\
& =\varphi_{*}\left(\nabla_{\dot{\gamma}}^{\lambda} \eta^{\perp}\right) \\
& =\varphi_{*} S(X, \eta)-\varphi_{*}\left(\nabla_{\dot{\gamma}}^{\lambda} \eta^{\top}\right) \\
& =\varphi_{*} S(X, \eta)+\sum_{i}\left\langle\eta^{\top}, T\left(X, e_{i}\right)\right\rangle_{M} \varphi_{*} e_{i} \\
& =\varphi_{*} S(X, \xi)+\sum_{i}\left\langle\xi, T\left(X, e_{i}\right)\right\rangle_{M} \varphi_{*} e_{i}
\end{aligned}
$$

and

$$
\pi_{*}\left(\Phi_{*} X_{\xi}^{h}\right)=\varphi_{*}\left(\pi_{*} X_{\xi}^{h}\right)=\varphi_{*} X
$$

the equality (1.11) holds.

By Lemma [1.7 we have (see also [1, Lemma 4.5.1]).

Corollary 1.8. (1) Let $X, Y \in \mathcal{H}_{x}^{\varphi}, \xi \in T_{x} M$. Then

$$
\begin{aligned}
B(X, \xi) & =\varphi_{*}(S(X, \xi))+P(X, \xi), \\
\left\langle B(X, \xi), \varphi_{*} Y\right\rangle_{N} & =\lambda\left(\langle S(X, \xi), Y\rangle_{M}+\langle\xi, T(X, Y)\rangle_{M}\right) .
\end{aligned}
$$

(2) Let $X \in \mathcal{H}_{x}^{\varphi}, \xi \in \mathcal{V}_{x}^{\varphi}$. Then

$$
\begin{aligned}
\left\langle\varphi_{*}(S(X, \xi)), P(X, \xi)\right\rangle_{N} & =0, \\
\left|B(X, \xi)_{0}^{v}\right|_{T N}^{2} & =\left|\left(\varphi_{*}(S(X, \xi))\right)_{0}^{v}\right|_{T N}^{2}+\left|P(X, \xi)_{0}^{v}\right|_{T N}^{2} .
\end{aligned}
$$

(3) $B(X, \xi)=0$ for all $X \in \Gamma\left(\mathcal{H}^{\varphi}\right), \xi \in \Gamma(T M)$ if and only if $T=0$ and $S=0$.

Proof. (1.12) follows by (1.11) and (1.4). For $X, Y \in \mathcal{H}_{x}^{\varphi}$ and $\xi \in T_{x} M$ we have

$$
\begin{aligned}
\left\langle B(X, \xi), \varphi_{*} Y\right\rangle_{N} & =\left\langle\varphi_{*}(S(X, \xi)), \varphi_{*} Y\right\rangle_{N}+\sum_{i}\left\langle\xi, T\left(X, e_{i}\right)\right\rangle_{M}\left\langle\varphi_{*} e_{i}, \varphi_{*} Y\right\rangle_{N} \\
& =\lambda\langle S(X, \xi), Y\rangle_{M}+\lambda\langle\xi, T(X, Y)\rangle_{M},
\end{aligned}
$$

which proves (1.13). Now, let $X \in \mathcal{H}_{x}^{\varphi}$ and $\xi \in \mathcal{V}_{x}^{\varphi}$. Then

$$
\varphi_{*}(S(X, \xi))=\frac{1}{2 \lambda}(\xi \lambda) \varphi_{*} X .
$$

Thus

$$
\begin{aligned}
\left\langle\varphi_{*}(S(X, \xi)), P(X, \xi)\right\rangle_{N} & =\frac{1}{2 \lambda}(\xi \lambda) \sum_{i}\left\langle\xi, T\left(X, e_{i}\right)\right\rangle_{M}\left\langle\varphi_{*} X, \varphi_{*} e_{i}\right\rangle_{N} \\
& =\frac{1}{2}\langle\xi, T(X, X)\rangle_{M}=0,
\end{aligned}
$$

since $T$ is skew-symmetric. Hence (1.14) holds, which implies (1.15). (3) is a consequence of $(\underline{1.12})$ and (1.14) . 
Let $X \in \mathcal{H}_{x}^{\varphi}$ and $\xi \in T_{x} M$. Decompose $S(X, \xi)_{\xi}^{v}$ into horizontal and vertical part with respect to $\mathcal{V}^{\Phi}$ and $\mathcal{H}^{\Phi}$,

$$
S(X, \xi)_{\xi}^{v}=S_{\Phi}^{\top}(X, \xi)+S_{\Phi}^{\perp}(X, \xi) .
$$

Equip tangent bundles $T M$ and $T N$ with Cheeger-Gromoll type metrics $h_{p, q, \alpha}$ and $h_{r, s, \beta}$, respectively.

Lemma 1.9. Assume $\varphi$ and $\Phi$ are both horizontally conformal with dilatations $\lambda$ and $\Lambda$, respectively. Let $X \in \mathcal{H}_{x}^{\varphi}, \xi \in T_{x} M$. Then

$$
\begin{aligned}
\Lambda(\xi)|X|_{M}^{2} & =\lambda(x)|X|_{M}^{2}+\left|B(X, \xi)_{\varphi_{*} \xi}^{v}\right|_{T N}^{2}, \\
\Lambda(\xi)|X|_{M}^{2}+\Lambda(\xi)\left|S_{\Phi}^{\perp}(X, \xi)\right|_{T N}^{2} & =\lambda(x)|X|_{M}^{2}+\left|P(X, \xi)_{\varphi_{*} \xi}^{v}\right|_{T N}^{2} .
\end{aligned}
$$

Proof. (1.16) follows directly by (1.4) (and Corollary 1.6). To prove (1.17), define $V=X_{\xi}^{h}-S(X, \xi)_{\varphi_{*} \xi}^{v}$. Then

$$
V+S_{\Phi}^{\top}(X, \xi)=X_{\xi}^{h}-S_{\Phi}^{\perp}(X, \xi) \in \mathcal{H}^{\Phi} .
$$

Therefore,

$$
\begin{aligned}
\left|\Phi_{*}\left(X_{\xi}^{h}-S_{\Phi}^{\perp}(X, \xi)\right)\right|_{T N}^{2} & =\Lambda(\xi)\left|X_{\xi}^{h}-S_{\Phi}^{\perp}(X, \xi)\right|_{T N}^{2} \\
& =\Lambda(\xi)|X|_{M}^{2}+\Lambda(\xi)\left|S_{\Phi}^{\perp}(X, \xi)\right|_{T N}^{2},
\end{aligned}
$$

since $\left\langle X_{\xi}^{h}, S_{\Phi}^{\perp}(X, \xi)\right\rangle_{T M}=\left\langle X_{\xi}^{h}, S(X, \xi)_{\xi}^{v}\right\rangle=0$, and, by (1.11),

$$
\begin{aligned}
\left|\Phi_{*}\left(V+S_{\Phi}^{\top}(X, \xi)\right)\right|_{T N}^{2} & =\left|\Phi_{*} V\right|_{T N} \\
& =\left|\left(\phi_{*} X\right)_{\varphi_{*} \xi}^{h}+P(X, \xi)_{\varphi_{*} \xi}^{v}\right|_{T N}^{2} \\
& =\lambda(x)|X|_{M}^{2}+\left|P(X, \xi)_{\varphi_{*} \xi}^{v}\right|_{T N}^{2} .
\end{aligned}
$$

Hence (1.17) holds.

Lemma 1.10. If $\Phi$ and $\varphi$ are both horizontally conformal, then $\lambda$ is constant.

Proof. Let $X \in \mathcal{H}^{\varphi}$ and $\xi \in \mathcal{V}^{\varphi}$. Comparing (1.16) and (1.17) and using (1.15) we get

$$
\left|S_{\Phi}^{\perp}(X, \xi)\right|_{T N}^{2}+\left|\left(\varphi_{*}(S(X, \xi))\right)_{0}^{v}\right|^{2}=0,
$$

which implies, $\varphi_{*}(S(X, \xi))=0$, so $\xi \lambda=0$. It follows that $\operatorname{grad} \lambda$ is horizontal.

Assume now, $\xi \in \mathcal{H}^{\varphi}$. Then $P(X, \xi)=0$. Again, comparing (1.16) and (1.17) we get

$$
0=\Lambda(\xi)\left|S_{\Phi}^{\perp}(X, \xi)\right|_{T N}^{2}+\left|B(X, \xi)_{\varphi_{*} \xi}^{v}\right|_{T N}^{2} .
$$

Thus

$$
B(X, \xi)=0 \quad \text { and } \quad S_{\Phi}^{\perp}(X, \xi)=0 .
$$


Hence, as before, $\varphi_{*} S(X, \xi)=0$. Since $\operatorname{grad} \lambda$ is horizontal, we may put $X=\xi=\operatorname{grad} \lambda$. Then

$$
0=\varphi_{*} S(\operatorname{grad} \lambda, \operatorname{grad} \lambda)=-\frac{1}{2 \lambda}|\operatorname{grad} \lambda|_{M}^{2} \varphi_{*}(\operatorname{grad} \lambda) .
$$

Therefore, $\operatorname{grad} \lambda$ is vertical. Finally, $\lambda$ is constant.

Lemma 1.11. Assume $\operatorname{dim} N \geq 2$. Let $\Phi$ be a horizontally conformal map with dilatation $\Lambda$. Then $\varphi$ is horizontally conformal with constant dilatation $\lambda=\Lambda(0)$. Moreover, $\Lambda$ is constant and the horizontal distribution $\mathcal{H}^{\varphi}$ is integrable.

Proof. Let $X, Y \in \mathcal{H}_{x}^{\varphi}, 0=0_{x} \in T_{x} M$. Then by Corollary [1.6, $X_{0}^{h}, Y_{0}^{h} \in$ $\mathcal{H}_{0}^{\Phi}$. By (1.4) $), \Phi_{*}\left(X_{0}^{h}\right)=\left(\varphi_{*} X\right)_{0}^{h}$ and $\Phi_{*}\left(Y_{0}^{h}\right)=\left(\varphi_{*} Y\right)_{0}^{h}$. Thus

$$
\Lambda(0)\langle X, Y\rangle_{M}=\left\langle\Phi_{*} X_{0}^{h}, \Phi_{*} Y_{0}^{h}\right\rangle_{T N}=\left\langle\varphi_{*} X, \varphi_{*} Y\right\rangle_{N} .
$$

Hence $\varphi$ is conformal with dilatation $\lambda(x)=\Lambda\left(0_{x}\right)$. By Lemma $1.10 \lambda$ is constant. Thus $S=0$.

Let $X, Y \in \Gamma\left(\mathcal{H}^{\varphi}\right), \xi \in \mathcal{V}^{\varphi}$. Then $X_{\xi}^{v}+\omega_{\alpha}(\xi)^{p}\left(\nabla_{\xi} X\right)_{\xi}^{h}, Y_{\xi}^{h} \in \mathcal{H}_{\xi}^{\Phi}$ by Theorem 1.5 and Corollary 1.6. Moreover, by Corollary 1.8

$$
\left\langle B(Y, \xi), \varphi_{*} X\right\rangle_{N}=\lambda\langle\xi, T(Y, X)\rangle_{M}
$$

Hence, by horizontal conformality of $\Phi$ and (1.9) we get

$$
\left(\Lambda(\xi)-\lambda-\lambda\left(1+\alpha|\xi|^{2}\right)^{p}\right)\langle\xi, T(Y, X)\rangle_{M}=\left\langle B\left(\nabla_{\xi}^{M} X, \xi\right), B(Y, \xi)\right\rangle_{N} .
$$

Put $A(\xi)=\Lambda(\xi)-\lambda$. Then by (1.16),$A(t \xi)=t^{2} A(\xi)$. Hence, replacing $\xi$ by $t \xi$ and assuming for simplicity $|\xi|_{M}=1$, we get

$t^{2}\left(A(\xi)\langle\xi, T(Y, X)\rangle_{M}-\left\langle B\left(\nabla_{\xi}^{M} X, \xi\right), B(Y, \xi)\right\rangle_{M}\right)=\lambda\left(1+\alpha t^{2}\right)^{p}\langle\xi, T(Y, X)\rangle_{M}$.

Therefore, $\langle\xi, T(Y, X)\rangle_{M}=0$ and, since $\xi \in \Gamma\left(\mathcal{V}^{\varphi}\right)$ was taken arbitrary, $T=0$. This implies, together with the fact that $S=0$ and Corollary 1.8 that

$$
B(X, \xi)=0 \text { for } X \in \mathcal{H}^{\varphi} \text { and } \xi \in T M .
$$

Hence, by (1.16),$\Lambda(\xi)=\lambda$ for any $\xi$.

\section{MAIN RESUlts}

2.1. Differential as a horizontally confomal map. Consider a smooth $\operatorname{map} \varphi:\left(M,\langle\cdot, \cdot\rangle_{M}\right) \rightarrow\left(N,\langle\cdot, \cdot\rangle_{N}\right), \operatorname{dim} M>\operatorname{dim} N \geq 2$, between Riemanian manifolds. Let $\Phi=\varphi_{*}:(T M, \tilde{h}) \rightarrow(T N, h)$, where $\tilde{h}=h_{p, q, \alpha}$ and $h=h_{r, s, \beta}$ are Cheeger-Gromoll type metrics. 
Theorem 2.1. $\Phi$ is horizontally conformal if and only if $\varphi$ is totally geodesic and horizontally conformal with constant dilatation $\lambda, p \alpha=r \beta=0$ and $q=\lambda s$. Then, the dilatation $\Lambda$ of $\Phi$ is constant and equal to $\lambda$, horizontal distribution $\mathcal{H}^{\varphi}$ is integrable and vertical distribution $\mathcal{V}^{\varphi}$ is totally geodesic.

Proof. Assume $\Phi$ is horizontally conformal. Then by Lemma 1.11, $\varphi$ is horizontally conformal with constant dilatation $\lambda$, the dilatation $\Lambda$ of $\Phi$ is constant and equal to $\lambda$ and by Corollary 1.8, $B(X, \xi)=0$ for $X \in \mathcal{H}^{\varphi}$ and $\xi \in T M$.

Let $X \in \Gamma\left(\mathcal{H}^{\varphi}\right)$ and $\xi \in \Gamma\left(\mathcal{V}^{\varphi}\right)$. Then $A=X_{\xi}^{v}+\omega_{\alpha}(\xi)^{p}\left(\nabla_{\xi}^{M} X\right)_{\xi}^{h} \in \mathcal{H}_{\xi}^{\Phi}$ by Theorem 1.5. Since $T=0$, by (1.9) $\nabla_{\xi}^{M} X \in \mathcal{V}^{\varphi}$. Then $\left|\Phi_{*} A\right|_{T N}^{2}=\lambda|A|_{T M}^{2}$ implies

$$
\begin{aligned}
\lambda|X|_{M}^{2}\left(1+\alpha|\xi|_{M}^{2}\right)^{p}\left(1-\left(1+\alpha|\xi|_{M}^{2}\right)^{p}\right)+\lambda\left|\nabla_{\xi}^{M} X\right|_{M}^{2} & \\
& =\left|B\left(\nabla_{\xi}^{M} X, \xi\right)\right|_{N}^{2}+2\left(1+\alpha|\xi|_{M}^{2}\right)^{p}\left\langle B\left(\nabla_{\xi}^{M} X, \xi\right), \varphi_{*} X\right\rangle_{N} .
\end{aligned}
$$

Replacing $\xi$ by $t \xi$, assuming $|\xi|_{M}^{2}=1$ and computing the second derivative at $t=0$, we get

$$
-\alpha p \lambda|X|_{M}^{2}+\lambda\left|\nabla_{\xi}^{M} X\right|_{M}^{2}=2\left\langle B\left(\nabla_{\xi}^{M} X, \xi\right), \varphi_{*} X\right\rangle_{N} .
$$

Since $\left\langle B\left(\nabla_{\xi} X, \xi\right), \varphi_{*} X\right\rangle_{N}=\lambda\left|\nabla_{\xi}^{M} X\right|_{M}^{2}$, we obtain $-p \alpha|X|_{M}^{2}=\left|\nabla_{\xi}^{M} X\right|_{M}^{2}$. Therefore, $p \alpha=0$ and $\nabla_{\xi}^{M} X=0$. This implies $B=0$ globally, so $\varphi$ is totally geodesic. In particular, horizontal distrubution is integrable and vertical distribution is totally geodesic.

Moreover, for $X \in \Gamma\left(\mathcal{H}^{\varphi}\right)$ basic and $\xi \in \mathcal{H}_{x}^{\varphi}$ such that $\xi$ and $X_{x}$ are orthogonal, by Theorem 1.5 and the fact that $\alpha p=0$ we have $A=X_{\xi}^{v}+$ $\left(\nabla_{\xi}^{M} X\right)_{\xi}^{h} \in \mathcal{H}_{\xi}^{\Phi}$. Since $\nabla_{\xi}^{M} X \in \mathcal{H}_{x}^{\varphi}$, the condition $\left|\Phi_{*} A\right|_{T N}^{2}=\lambda|A|_{T M}^{2}$ simplfies to

$$
\omega_{\beta}\left(\varphi_{*} \xi\right)^{r}=1
$$

This implies $r \beta=0$. Now, for arbitrary $X, \xi \in \Gamma\left(\mathcal{H}^{\varphi}\right), A=X_{\xi}^{v}-$ $q \omega_{q}(\xi)\langle X, \xi\rangle \xi_{\xi}^{v}+\left(\nabla_{\xi}^{M} X\right)_{\xi}^{h} \in \mathcal{H}^{\Phi}$. Then, as above by horizontal conformality of $\Phi$, after some computations

$$
q+q^{3} \omega_{q}(\xi)^{2}|\xi|_{M}^{4}-2 q^{2} \omega_{q}(\xi)|\xi|_{M}^{2}=\lambda s+\lambda s q^{2} \omega_{q}(\xi)^{2}|\xi|_{M}^{2}-2 \lambda s q \omega_{q}(\xi)|\xi|_{M}^{2}
$$

Hence $q=\lambda s$.

Conversely, assume $\varphi$ is totally geodesic and horizontally conformal with constant dilatation $\lambda, p \alpha=r \beta=0$ and $q=\lambda s$. By Theorem 1.5 $\Phi$ : $T M \rightarrow T N$ is a submersion and vertical and horizontal distributions $\mathcal{V}^{\Phi}$ and $\mathcal{H}^{\Phi}$ are given by (1.5) and (1.6), respectively. It remains to show that 
$\Phi_{*}: \mathcal{H}^{\Phi} \rightarrow T(T N)$ is conformal, but this follows immediately by simple calculations.

2.2. Differential as a harmonic morphism. Let $\varphi:\left(M,\langle\cdot, \cdot\rangle_{M}\right) \rightarrow$ $\left(N,\langle\cdot, \cdot\rangle_{N}\right)$. Consider notation from the first section. We say that $\varphi$ is harmonic if its tension field $\tau(\varphi)=\operatorname{tr} B$ vanishes. If $e_{1}, \ldots, e_{n}$ is an orthonormal frame on $M$ then

$$
\tau(\varphi)=\sum_{i}\left(\nabla_{e_{i}}^{\varphi} \varphi_{*} e_{i}-\varphi_{*}\left(\nabla_{e_{i}}^{M} e_{i}\right)\right)
$$

A map $\varphi: M \rightarrow N$ is said to be a harmonic morphism if for every harmonic function $f: G \rightarrow \mathbb{R}$ defined on an open subset $G$ of $N$ with $\varphi^{-1}(G)$ nonempty, the composition $f \circ \varphi$ is a harmonic map on $\varphi^{-1}(G)$. There is a useful characterisation of harmonic morphisms due to Fuglede and Ishihara. Namely, see [1, $\varphi$ is a harmonic morphisms if and only if it is horizontally conformal and harmonic. Moreover, if $\varphi$ is horizontally conformal, then its tension field simplifies to [1]

$$
\tau(\varphi)=-\frac{n-2}{2} \varphi_{*}(\operatorname{grad}(\ln \lambda))-(m-n) \varphi_{*}\left(\kappa_{\varphi}\right),
$$

where $m=\operatorname{dim} M, n=\operatorname{dim} N$ and $\kappa_{\varphi}$ is the mean curvature vector field of the fibres of $\varphi$,

$$
\left.\kappa_{\varphi}=\frac{1}{m-n} \sum_{i}\left(\nabla_{e_{i}}^{M} e_{i}\right)\right)^{\perp}
$$

where $e_{1}, \ldots, e_{m-n}$ is an orthonormal frame of $\mathcal{V}^{\varphi}$.

Equip tangent bundle $T M$ with Cheeger-Gromoll type metric $h_{p, q, \alpha}$. Let $\omega_{q}(\xi)=\left(1+q|\xi|_{M}^{2}\right)^{-1}$. The Levi-Civita connection $\nabla^{T M}$ corresponding to $h_{p, q, \alpha}$ evaluated at $\xi$ is given by [8]

$$
\begin{aligned}
\nabla_{X^{h}}^{T M} Y^{h} & =\left(\nabla_{X} Y\right)^{h}-\frac{1}{2}(R(X, Y) \xi)^{v} \\
\nabla_{X^{h}}^{T M} Y^{v} & =\left(\nabla_{X} Y\right)^{v}+\frac{1}{2} \omega(\xi)^{p}(R(\xi, Y) X)^{h} \\
\nabla_{X^{v}}^{T M} Y^{h} & =\frac{1}{2} \omega(\xi)^{p}(R(\xi, X) Y)^{h} \\
\nabla_{X^{v}}^{T M} Y^{v} & =-\alpha p \omega(\xi)\left(\langle X, \xi\rangle_{M} Y+\langle Y, \xi\rangle_{M} X\right)^{v} \\
& +(\alpha p \omega(\xi)+q) \omega_{q}(\xi)\langle X, Y\rangle_{M} \xi_{\xi}^{v}+\alpha p q \omega(\xi) \omega_{q}(\xi)\langle X, \xi\rangle_{M}\langle Y, \xi\rangle_{M} \xi_{\xi}^{v}
\end{aligned}
$$

Let $\varphi:\left(M,\langle\cdot, \cdot\rangle_{M}\right) \rightarrow\left(N,\langle\cdot, \cdot\rangle_{N}\right), \operatorname{dim} M>\operatorname{dim} N \geq 2$, be a smooth map between Riemanian manifolds, $\Phi=\varphi_{*}:(T M, \tilde{h}) \rightarrow(T N, h)$, where $\tilde{h}=h_{p, q, \alpha}$ and $h=h_{r, s, \beta}$ are Cheeger-Gromoll type metrics.

Theorem 2.2. $\Phi$ is a harmonic morphism if and only if $\varphi$ is a totally geodesic harmonic morphism, the dilatation of $\varphi$ is constant and $\tilde{h}, h$ are Sasaki metrics. 
Proof. Assume $\Phi$ is a harmonic morphism. In particular, $\Phi$ is horizontally conformal. Hence, by Theorem 2.1, $\varphi$ is horizontally conformal, the dilatations of $\Phi$ and $\varphi$ are constant, $\Lambda=\lambda$, and $\varphi$ is totally geodesic. Therefore $\varphi$ is harmonic, so $\varphi$ is a harmonic morphism. Thus, by (2.2) $, \kappa_{\varphi}=0, \kappa_{\Phi}=0$. Moreover, by Theorem 2.1, $p \alpha=r \beta=0$ and $q=\lambda s$. Let $\xi \in \mathcal{H}^{\varphi}$ and let $e_{1}, \ldots, e_{m-n}$ be an orthonormal frame for $\mathcal{V}_{\xi}^{\varphi}, m=\operatorname{dim} M, n=\operatorname{dim} N$. Since vertical distribution $\mathcal{V}^{\varphi}$ is, by Theorem 2.1 totally geodesic, by Theorem 1.5 and Corollary 1.6

$$
E_{i}=\left(e_{i}\right)_{\xi}^{h}, \quad F_{i}=\left(e_{i}\right)_{\xi}^{v}, \quad i=1, \ldots, m-n,
$$

is an orthonormal frame for $\mathcal{V}_{\xi}^{\Phi}$. Then

$$
\begin{aligned}
\nabla_{E_{i}}^{T M} E_{i} & =\left(\nabla_{e_{i}}^{M} e_{i}\right)_{\xi}^{h}, \\
\nabla_{F_{i}}^{T M} F_{i} & =q \omega_{q}(\xi) \xi_{\xi}^{v} .
\end{aligned}
$$

Therefore,

$$
\Phi_{*}\left(\kappa_{\Phi}\right)=\frac{1}{2}\left(\varphi_{*}\left(\kappa_{\varphi}\right)\right)_{\varphi_{*} \xi}^{h}+\frac{1}{2} q \omega_{q}(\xi)\left(\varphi_{*} \xi\right)_{\varphi_{*} \xi}^{v}
$$

Since $\Phi_{*}\left(\kappa_{\Phi}\right)=0$ and $\varphi_{*}\left(\kappa_{\varphi}\right)=0$, it follows that $q=0$. Hence, $s=0$ and $\tilde{h}, h$ are Sasaki metrics.

Conversely, assume $\varphi$ is a totally geodesic harmonic morphism, the dilatation of $\varphi$ is constant and $\tilde{h}, h$ are Sasaki metrics. Then, by Theorem 2.1 $\Phi$ is horizontally conformal with constant dilatation. Moreover, $\varphi_{*}\left(\kappa_{\varphi}\right)=0$. Hence, similarly as above,

$$
\Phi_{*}\left(\kappa_{\Phi}\right)=\frac{1}{2}\left(\varphi_{*}\left(\kappa_{\varphi}\right)\right)^{h}=0,
$$

and by (2.2), $\Phi$ is a harmonic morphism.

Remark 2.3. Condition $p \alpha=0$ for Cheeger-Gromoll metric $h_{p, q, \alpha}$ is equavalent to condition $p=0$ for the metric $h_{p, q}$. It follows that in the case of horizontal conformality of a differential, and in consequence for a differential to be a harmonic morphism, it is sufficient to consider only $h_{p, q}$ metrics introduced in [3].

\section{REFERENCES}

[1] P. Baird, J. C. Wood, Harmonic morphisms between Riemannian manifolds, Oxford University Press, Oxford 2003.

[2] M. Benyounes, E. Loubeau, C. M. Wood, Harmonic sections of Riemannian vector bundles, and metrics of Cheeger-Gromoll type, Differential Geom. Appl. 25 (2007), no. 3, 322-334.

[3] M. Benyounes, E. Loubeau, C. M. Wood, The geometry of generalised CheegerGromoll metrics, preprint, arXiv:math/0703059.

[4] A.L. Besse, Einstein Manifolds, Springer-Verlag, Berlin, Heidelberg, 1987. 
[5] P. Dombrowski, On the geometry of the tangent bundle, J. Reine Angew. Math. 210 (1962), 73-88.

[6] W. Kozłowski, K. Niedziałomski, Conformality of a differential with respect to Cheeger-Gromoll type metrics, preprint, arXiv:0809.4427.

[7] W. Kozłowski, Sz. M. Walczak, Collapse of unit horizontal bundles equipped with a metric of Cheeger-Gromoll type, Differential Geom. Appl. 27 (2009), no. 3, 378-383.

[8] M. I. Munteanu, Cheeger-Gromoll type metrics on the tangent bundle, Sci. Ann. Univ. Agric. Sci. Vet. Med. 49 (2006), no. 2, 257-268.

Department of Mathematics and Computer Science

UNIVERSITY OF ŁÓDŹ

UL. BANACHA 22, 90-238 ŁÓDŹ

POLAND

E-mail address: wojciech@math.uni.lodz.pl

Department of Mathematics and Computer Science

UNIVERSITY OF ŁÓDŹ

UL. BANACHA 22, 90-238 ŁÓDŹ

POLAND

E-mail address: kamiln@math.uni.lodz.pl 